\title{
Operator content of entanglement spectra in the transverse field Ising chain after global quenches
}

\author{
Jacopo Surace, ${ }^{1,2}$ Luca Tagliacozzo, ${ }^{1,3}$ and Erik Tonni ${ }^{4}$ \\ ${ }^{1}$ Department of Physics and SUPA, University of Strathclyde, Glasgow G4 ONG, United Kingdom \\ ${ }^{2}$ ICFO-Institut de Ciències Fotóniques, The Barcelona Institute of Science and Technology, 08860 Castelldefels (Barcelona), Spain \\ ${ }^{3}$ Departament de Física Quàntica i Astrofísica and Institut de Ciències del Cosmos (ICCUB), \\ Universitat de Barcelona, Martí i Franquès 1, 08028 Barcelona, Catalonia, Spain \\ ${ }^{4}$ SISSA and INFN Sezione di Trieste, via Bonomea 265, 34136 Trieste, Italy
}

(Received 4 October 2019; revised manuscript received 7 May 2020; accepted 19 May 2020; published 8 June 2020)

\begin{abstract}
We consider the time evolution of the gaps of the entanglement spectrum for a block of consecutive sites in finite transverse field Ising chains after sudden quenches of the magnetic field. We provide numerical evidence that, whenever we quench at or across the quantum critical point, the time evolution of the ratios of these gaps allows us to obtain universal information. They encode the low-lying gaps of the conformal spectrum of the Ising boundary conformal field theory describing the spatial bipartition within the imaginary time path integral approach to global quenches at the quantum critical point.
\end{abstract}

DOI: 10.1103/PhysRevB.101.241107

\section{INTRODUCTION}

Quantum many-body systems described by local Hamiltonians are difficult to solve because the dimensionality of their Hilbert space increases exponentially with the number of their constituents. At equilibrium however the wave functions of quantum many-body systems characterized by gapped Hamiltonians contain a limited amount of entanglement with a well defined structure that can be used to perform numerical simulations with tensor networks [1-6]. At quantum critical points (QCPs), where the Hamiltonians become gapless, the ground state wave function becomes more complex, since the entanglement grows. This growth follows universal laws [7] that allow us to unveil the data of the underlying conformal field theory (CFT) in the specific case of conformal invariant QCPs [8,9]. In two spacetime dimensions, these data include the central charge $c$, the exponents dictating the decay of correlation functions (the conformal spectrum), and the coefficients of the operator product expansion.

The power of universality allows us to obtain the universal data by studying the system, e.g., by discretizing the space on a finite lattice and performing finite-size scaling [10], or by limiting the amount of entanglement in the ground state and performing finite-entanglement scaling [11]. In two spacetime dimensions, the central charge $c$ can be read from the scaling of the entanglement entropy (EE) of an interval with respect to its size [12-15]. The remaining CFT data are encoded, e.g., in the $\mathrm{EE}$ of a bipartition where a part is made by disjoint intervals [16-20], in a complicated way. The entanglement spectrum (ES) [21-30], i.e., the spectrum of the reduced density matrix, can be related to the conformal spectrum of a conformal field theory with boundaries (BCFT) defined by proper conformal boundary conditions (CBC) [31], as also observed by Läuchli in numerical studies [30].

Entanglement plays a central role in our understanding of large many-body quantum systems; indeed it gives access to universal information without needing to understand the details of the model, such as, e.g., the presence of an order parameter. As a result, numerous proposals on how to measure entanglement in experiments have led toexperimental measures in the context of cold atoms and trapped ions both at and out of equilibrium [32-43]. Experiments are expected to play an increasingly important role out of equilibrium [35], where the amount of entanglement increases to its maximum value very fast with time [44-46]. This entanglement barrier prevents one from extracting reliable predictions at long time even when our best classical-simulation techniques are employed [47-50] (see Refs. [51-55] for recent proposals to overcome this barrier). As a result, important questions about strongly correlated many-body quantum systems out of equilibrium are only partially understood and currently can only be settled experimentally. Examples of open questions include the existence of phases of matter out of equilibrium [56-58], the presence/absence of equilibration, the phenomenon of thermalization or generalized thermalization that should reconcile the reversibility of quantum mechanics with the irreversibility of the macroscopic world [59-62].

While exact solutions of the out-of-equilibrium dynamics are exponentially expensive, several approximate methods are available. They include semiclassical approximations based of truncated Wigner distributions, spin-wave analysis, and tensor network simulations where the dynamics is restricted on lowentangled subspace of the Hilbert space.

It is important to understand if those approximate solutions retain information about relevant physical properties of the dynamics. Equilibrium phenomena seem to suggest that, if a form of universality out of equilibrium is identified, it should be detectable even through these approximate methods, after appropriate scaling analysis. In this letter we discuss a form of universality based on out-of-equilibrium dynamics.

Out-of-equilibrium universality has been observed only in some specific scenarios where the scaling of the 
spatio-temporal evolution of the system can be described by universal exponents and functions (see Ref. [63] for a recent experiment and references). Dynamical quantum phase transitions, appearing as singularities in the return probabilities to the initial state quenched across a quantum critical point, could also provide a scaling region explainable through outof-equilibrium universality [64-66].

In this paper we consider the spin- $\frac{1}{2}$ transverse field Ising chain (TFIC). We study the time evolutions of the ES of an interval made by consecutive sites after a global quench. We provide numerical evidence that, for quenches to the QCP or across the QCP, the gaps of the ES carry information about the conformal spectrum of the Ising BCFT describing the QCP. These results are quite robust under changes of the quench protocol, suggesting an example of out-of-equilibrium universality.

\section{SETUP}

The Hamiltonian of the TFIC is

$$
H(\theta)=-\frac{1}{2}\left(\sum_{i=1}^{L-1} \sigma_{i}^{x} \sigma_{i+1}^{x}+\cot \theta \sum_{i=1}^{L} \sigma_{i}^{z}+\eta \sigma_{L}^{x} \sigma_{1}^{x}\right),
$$

where $L$ is the number of sites, $\sigma_{i}^{\alpha}$ are the Pauli matrices at the $i$-th site, and $\cot \theta$ is the magnetic field, with $0<\theta<$ $\frac{\pi}{2}$. The parameter $\eta$ encodes the boundary conditions: here we consider $\eta=1$ or $\eta=0$, corresponding, respectively, to periodic boundary conditions (PBC) or free open boundary conditions $(\mathrm{OBC})$. At zero temperature, this model exhibits a ferromagnetic (ordered) phase for $\frac{\pi}{4}<\theta<\frac{\pi}{2}$ and a paramagnetic (disordered) phase for $0<\theta<\frac{\pi}{4}$, separated by a QCP at $\theta=\frac{\pi}{4}$.

The bipartite entanglement can be studied by partitioning the system into an interval $A$ made by $\ell<L / 2$ consecutive sites and its complement $B$. Introducing the reduced density matrix $\rho_{A}=\operatorname{Tr}_{B} \rho$, we study its eigenvalues $\lambda_{i} \equiv e^{-\xi_{i}}$, where $\xi_{i} \geqslant 0$, which provide the ES. The EE of $A$ is $S_{A}=$ $-\operatorname{Tr}_{A}\left(\rho_{A} \log \rho_{A}\right)$. The system is prepared in the ground state $\left|\psi_{0}\right\rangle$ of Eq. (1) with $\theta=\theta_{0}$. At $t=0$, the quench $\theta_{0} \rightarrow \theta$ of the magnetic field brings the system out of equilibrium and the time evolved state for $t>0$ is $|\psi(t)\rangle=e^{-\mathrm{i} H(\theta) t}\left|\psi_{0}\right\rangle$. It is worth considering the gaps $g_{r} \equiv \log \lambda_{\max }-\log \lambda_{r}=$ $\xi_{r}-\xi_{\min } \geqslant 0$ with $r \geqslant 1$ and also their ratios $g_{r} / g_{s}$, that are independent of an arbitrary shift of the eigenvalues and of a rescaling of the entire spectrum.

We study the time evolutions of $g_{r} / g_{1}$ and of $\left(\ell g_{r}\right)^{-1}$ after quenches of $\theta$ in the TFIC for the first 16 eigenvalues ( $g_{r} S_{A}$ has been also considered in Ref. [67]). The numerical results are obtained by using the mapping of the TFIC to free fermions [68-72] (see also Ref. [53] and references therein). Similar studies have been recently performed in an infinite harmonic chain and an infinite chain of free fermions at half filling [73].

When the subsystem $A$ is half of an infinite line, it has been found through a CFT analysis that the time evolution after a global quench to the QCP of $g_{r} / g_{s},\left(\ell g_{r}\right)^{-1}$ and $g_{r} S_{A}$ encodes the critical exponents of the underlying BCFT [31]. The corresponding analyses for the configurations considered in this paper are not yet available in the literature.

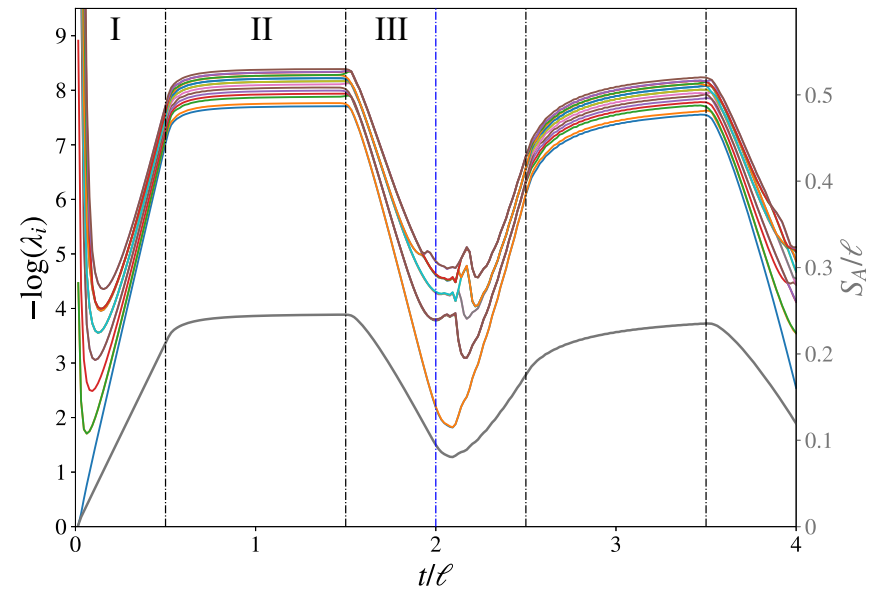

FIG. 1. Time evolutions of the first 16 eigenvalues $\lambda_{\max } \geqslant \lambda_{1} \geqslant$ $\lambda_{2} \geqslant \cdots \geqslant \lambda_{15}$ of the ES and of the EE (grey line) for an interval with $\ell=64$ sites in the chain with $L=256$ sites and PBC after the quench $\theta=\pi / 8 \rightarrow \theta=\pi / 4$ to the QCP. Different degeneracies are observed in regimes I, II, and III within the period.

\section{QUENCHES AT THE QCP}

We show the results only for the quench $\theta_{0}=\pi / 8 \rightarrow \theta=$ $\pi / 4$, from the ferromagnetic phase to the QCP, because other quenches provide similar outcomes. The subsystem $A$ is a block of a chain with PBC (Figs. 1 and 2) or a block at the beginning of a chain with $\mathrm{OBC}$ (Fig. 3). The quasiparticle picture $[44,74,75]$ allows us to identify different regimes in the time evolutions of the ES and of the EE, which are separated by black dashed-dotted vertical lines in the figures. After the quench, pairs of entangled quasiparticles are emitted from every site in opposite directions with velocity $v_{\mathrm{q}}=$ $\min (1, \cot \theta)[76,77]$. This leads to a linear increase while the initial quasi particles are still crossing the subsystem (regime I) and then to a saturation when their flux becomes stationary (regime II). In finite systems we also observe recurrences: the quasiparticles emitted at the same point meet periodically at times that are multiple integers of $\frac{L / 2}{v_{q}}$ for $\mathrm{PBC}$ and of $\frac{L}{v_{\mathrm{q}}}$ for OBC (blue dashed-dotted vertical lines) [78-81]. For PBC (Figs. 1, 2 and 4), we can identify three regimes I, II, III within a period, ending, respectively, at $t_{1}=\frac{\ell / 2}{v_{\mathrm{q}}}$ (equilibration time), $t_{2}=\frac{(L-\ell) / 2}{v_{\mathrm{q}}}$ and $t_{3}=\frac{L / 2}{v_{\mathrm{q}}}$, while for OBC (Fig. 3) these times are doubled.

In the time evolutions shown in Figs. 2 and 3, $\left(\ell g_{r}\right)^{-1}$ (top panels) roughly grow linearly, stay constant or decrease linearly in regime I, II, and III, respectively, like the EE.

Analytic CFT expressions for the time evolutions of the ES in Figs. 2 and 3 are not available in the literature. Nonetheless, some of their characteristic features can be understood through the CFT analysis performed in Ref. [31] for the ES of a semi-infinite half of the infinite line, based on BCFT [82,83].

The continuum limit of the TFIC at the QCP is described by the Ising CFT, whose central charge is $c=\frac{1}{2}$. In the presence of boundaries, the conformal symmetry of the Ising BCFT allows only three CBC. By adopting the BCFT approach to global quantum quenches with critical evolution Hamiltonians [44,74,75,78,79] (see Ref. [84] for a recent 


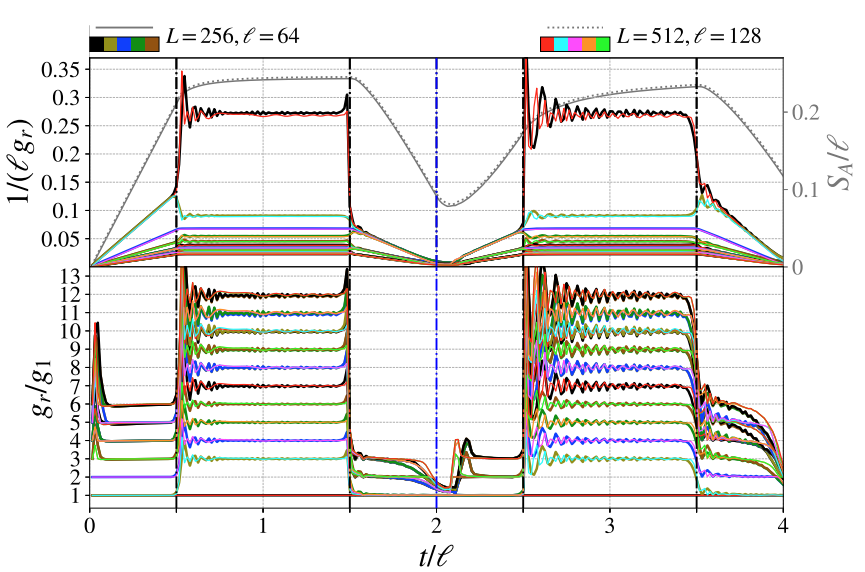

FIG. 2. Time evolution of $\left(\ell g_{r}\right)^{-1}$ (top), $g_{r} / g_{1}$ (bottom) after the quench $\theta_{0}=\pi / 8 \rightarrow \theta=\pi / 4$ for an interval in the chain with PBC.

review) and following Ref. [31] (see also Refs. [12,85-89]), we regularize the ultraviolet (UV) divergencies by removing small disks, with radius given by the infinitesimal UV cutoff, centered at the entangling points. This introduces a boundary around each entangling point, where a CBC $a$ must be imposed (see Ref. [67] for more details about the BCFT analysis). When the subsystem is half of the infinite line, in Ref. [31] it was found that the spacetime can be conformally mapped into an annulus with different CBC $a$ and $b_{0}$ (the latter one is related to the initial state [44]). This mapping allows us to obtain that $1 / g_{r}=\left(2 \pi \tau_{0} \Delta_{r}\right)^{-1} t$ grow linearly in time and that $g_{r} / g_{1}=\Delta_{r} / \Delta_{1}$ are independent of time, where $r \geqslant 1$ and $\Delta_{r} \in \mathscr{S}\left(a, b_{0}\right) \backslash\{0\}$ are the nonvanishing conformal dimensions of the primary fields and of their descendants of the BCFT on the annulus with CBC $a$ and $b_{0}$. The parameter $\tau_{0}$ encodes information about the initial state [90]. We remark that also $g_{r} S_{A}$ provides the conformal spectrum of the underlying BCFT [67].

When the system is finite, like in this manuscript, and the subsystem $A$ has $N$ entangling points $(N$ must be even for PBC), the spacetime has the topology of a sphere with $N+2$ boundaries for PBC and with $N+1$ boundaries for OBC. In the Euclidean spacetime, the $\mathrm{CBC} b_{1}$ and $b_{2}$ are imposed along the parallel segments corresponding to the physical boundaries (for the OBC that we consider $b_{1}=b_{2} \equiv b$, given by free boundary conditions).

At the beginning (regime I), we expect that the time evolution of the ES is determined only by the spacetime around the entangling points (some arguments are given in Ref. [67]). This assumption leads to consider $N$ independent copies of the spacetime corresponding to the time evolution of half of the infinite line; hence to employ the conformal spectrum of $N$ independent copies of the BCFT on the annulus found for the semi-infinite line. Combining this assumption with the results of Ref. [83] and assigning free boundary conditions both for the CBC $a$ (as found also through the numerical analysis of the ES for some bipartitions at equilibrium [30]) and for the CBC $b_{0}$, we can explain the data in the regimes I of Figs. 2 and 3, where $N=2$ and $N=1$, respectively. We remark that also $b$ corresponds to free boundary conditions in our numerical analysis. These assignments lead to $\mathscr{S}\left(a, b_{0}\right)=\mathscr{S}(a, a)=$ $\mathscr{S}(a, b)=\left\{0, \frac{1}{2}, \frac{3}{2}, 2, \frac{5}{2}, 3, \frac{7}{2}, 4, \frac{9}{2}, 5, \ldots\right\}$ (for recent numer-

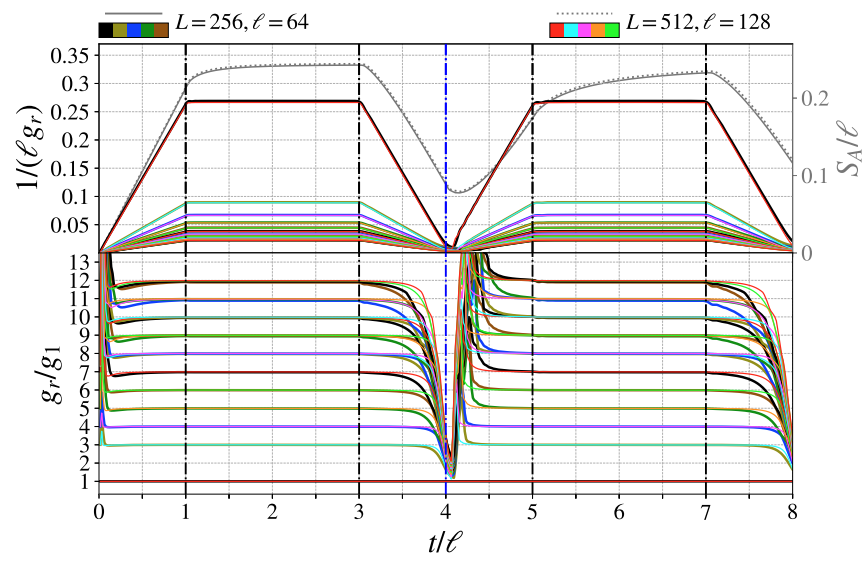

FIG. 3. Time evolution of $\left(\ell g_{r}\right)^{-1}$ (top), $g_{r} / g_{1}$ (bottom) after the quench $\theta_{0}=\pi / 8 \rightarrow \theta=\pi / 4$ for an interval in the chain with OBC.

ical results see Refs. [91,92]), which implies that $\mathscr{S}_{N}\left(a, b_{0}\right)=$ $\left\{0, \frac{1}{2}, 1, \frac{3}{2}, 2, \frac{5}{2}, 3, \frac{7}{2}, 4, \frac{9}{2}, 5, \ldots\right\}$ for any $N$.

The quasiparticle picture allows to argue that the regimes II are not influenced by the finite size of the system for the chosen bipartitions, where $\ell<L / 2$. It also identifies the boundaries of the finite Euclidean spacetime that presumably influence the time evolutions in this time regime. For all the chosen bipartitions we just need the conformal spectrum of a single Ising BCFT on the annulus. In particular, for Fig. $2 \mathscr{S}(a, a)$ has to be considered because the relevant boundaries are the infinitesimal circles around the entangling points. Instead, in Fig. 3 the physical boundary becomes important in regime II and $\mathscr{S}(a, b)$ has to be employed (see also Ref. [89] for this case). In our numerical analysis both $a$ and $b$ correspond to free boundary conditions, hence they are not distinguishable. These observations lead to expect that all the regimes II are identical and this is confirmed in Figs. 2 and 3, up to oscillations.

Summarizing, the time evolutions of $g_{r} / g_{1}$ when $0<\theta_{0}<$ $\pi / 4$, for the bipartitions considered, seem determined by the gaps of the conformal spectrum given by $\left(0 \oplus \frac{1}{2}\right)^{N}$ in regime I and by $0 \oplus \frac{1}{2}$ in regime II. The above considerations based on BCFT are expected to hold only for the low-lying part of the ES [93].

\section{QUENCHES TO GAPPED HAMILTONIANS}

By quenching across the QPT of the Ising model we encounter a dynamical quantum phase transition [64]. A dynamical quantum phase transition occurs at times when the return probability to the original state after the quench vanishes (see [66] for a recent review). Usual quantum phase transitions induce a universal behavior of correlation functions. Although the return probability to the ground state bares similarities with a boundary partition function at complex temperature, a dynamical quantum phase transition does not lead to the same universal features for the time-dependent correlation functions.

We find that the entanglement spectrum provides universal information also crossing the dynamical quantum phase transitions. Figure 4 shows the outcomes for a typical quench across the QCP: qualitatively different ES are observed when 


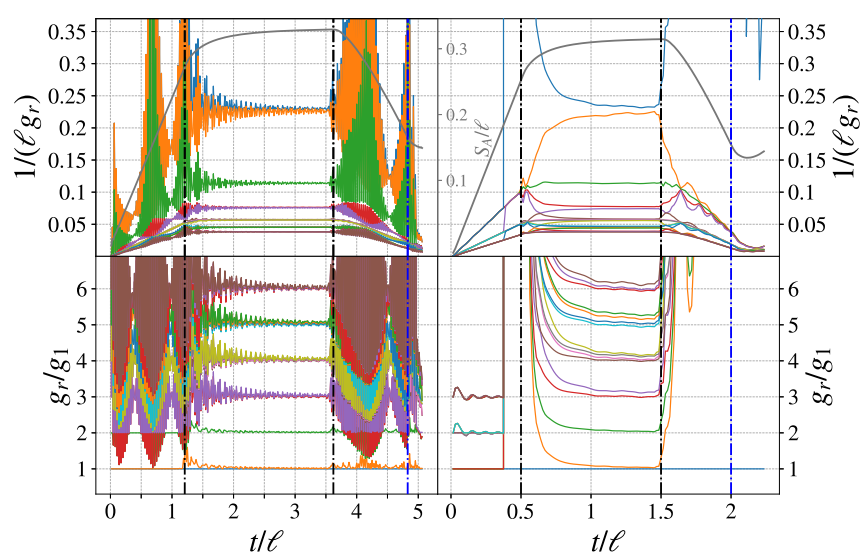

FIG. 4. Time evolution of $\left(\ell g_{r}\right)^{-1}$ (top), $g_{r} / g_{1}$ (bottom) after quenches such that $H$ and $H_{0}$ belong to different phases given by $\theta_{0}=\pi / 8 \rightarrow \theta=\pi / 2-\pi / 8$ (left) and $\theta_{0}=\pi / 2-\pi / 8 \rightarrow \theta=$ $\pi / 8$ (right), for an interval having $\ell=128$ sites in the chain with PBC having $L=512$ sites.

the two different directions between the phases are considered. In regimes I and III of the quench having $\theta_{0}<\frac{\pi}{4}$ and $\theta>\frac{\pi}{4}$ (Fig. 4, left panels), we observe spikes for the ratios $g_{r} / g_{1}$ : they identify the points in time where the first gap vanishes and hence the ratios diverge. In regime I, these times coincide with the times when the Loschmidt echo is singular [94]. This implies that these are the times that identify the location of dynamical quantum phase transitions.

For quenches across the QCP in the other direction (Fig. 4, right panels), the spikes are not observed. Still the ES becomes gapless, as witnessed by the linear increase of the inverse gaps in the regimes I of the quenches in both directions (modulo the spikes discussed above) [94,95]. In quenches from larger to smaller $\theta$, this is more visible due to the absence of spikes. The vanishing of the gap in the entanglement spectrum already observed for quenches at the critical point is specific to quenches across a dynamical quantum phase transition. For generic quenches in the same phase the entanglement spectrum indeed remains gapped as we show in Ref. [67].

The ratios $g_{r} / g_{1}$ display surprising behaviors, allowing us to identify the gapless spectrum of the QCP. Indeed, in the right panel of Fig. 4 they approach integer values in regimes I and II. Furthermore, similar plateaux at integer values are observed also in regime II in the left panel. This is the same feature discussed above for the quenches at the QCP (see regime I in Fig. 2) and could be attributed to the crossing of the QCP. This hypothesis is supported by the fact that, when the QCP is not crossed, the same plateaux at integer values for $g_{r} / g_{1}$ in regime II are not observed (see Fig. 2 of Ref. [67]).

The collapse of the curves of $g_{r} / g_{1}$ on the integer values in regime II for the quench from the paramagnetic phase to the ferromagnetic phase improves as $\left|\theta-\theta_{0}\right|$ increases. According to the BCFT discussion relevant for the quenches at the QCP, these integer values could be related to two copies of the Ising BCFT on the annulus with free boundary conditions, like in regime I of the quench at the QCP (see Fig. 2). A possible interpretation could rely on the fact that, being the evolution Hamiltonian gapped, the correlation length is finite; hence the entangling points can be considered independent from each other, as in regime I of the quench at the QCP. It would be important to support these observations through analytic results.

The possibility of defining scaling and universality when crossing several dynamical quantum phase transitions is still debated [66]. Our results seem to indicate that this is the case when the entanglement spectrum is considered.

\section{SUMMARY AND DISCUSSION}

In finite TFIC with either PBC or OBC (reflecting boundaries), we studied numerically the time evolution of the gaps $g_{r}$ of the ES of a block after a global quantum quench of the magnetic field. Interesting information is provided by the ratios $g_{r} / g_{1}$, which take constant values in various time regimes, for the lower part of the ES. When we quench to the QCP, the first thermalization regime (regime II) is determined by the conformal spectrum of the Ising BCFT with the proper CBC. Surprisingly, this feature is observed also for quenches across the QCP, where it provides insight on the possible presence of universality and scaling. Furthermore, these results are robust under reasonable changes of the initial state. Our analysis leads also to identify the proper CBC to adopt in the BCFT approach to global quenches $[31,44,74,75,78,79]$ that allow us to reproduce the numerical results (as done in Ref. [30] at equilibrium in the ground state).

This work can be extended by considering different boundary conditions, different quench protocols [96], different bipartitions and quenches in interacting spin chains (e.g., $X X Z$ and the Hubbard model). It is important to understand quantitatively the role of the generalized Gibbs ensemble in our analysis. Some preliminary results in this direction are presented in Ref. [67]. The relation between the ES after a quench across the QCP and the features of the dynamical quantum phase transitions also deserves further, more quantitative, analysis. Interesting results about the ES could be obtained also by considering directly the full entanglement Hamiltonian [73,85,97-105], even for spatially inhomogeneous lattice systems [88]. Extending all these analyses to higher dimensions is also very important. Our results naturally suggest that experiments on entanglement spectroscopy of out-of-equilibrium correlated many-body quantum systems could provide ways to measure the critical exponents.

\section{ACKNOWLEDGMENTS}

We are grateful to G. Di Giulio, G. Evenbly, A. Ludwig, G. Mussardo, A. Polkovnikov and M.s Rigol for useful discussions and in particular to J. Cardy for his comments on the draft. J.S. is supported by the doctoral training partnership (DTP 2016-2017) of the University of Strathclyde. L.T. is supported by the MICINN RYC-2016-20594 fellowship and the MICINN PGC2018-095862-B-C22 grant. We thank the organizers of the workshop Entangle This IV: Chaos, Order and Qubits (ICMAT, Madrid), where these results have been presented by L.T. E.T. acknowledges Yukawa Institute for Theoretical Physics at Kyoto University for kind hospitality during the workshop YITP-T-19-03 Quantum Information and String Theory 2019 and Trieste Institute for Theoretical Quantum Technologies. 
[1] M. B. Hastings, J. Stat. Mech.: Theory Exp. (2007) P08024.

[2] L. Masanes, Phys. Rev. A 80, 052104 (2009).

[3] L. Amico, R. Fazio, A. Osterloh, and V. Vedral, Rev. Mod. Phys. 80, 517 (2008).

[4] J. C. Bridgeman and C. T. Chubb, J. Phys. A 50, 223001 (2017).

[5] R. Orús, Ann. Phys. (NY) 349, 117 (2014).

[6] S.-J. Ran, E. Tirrito, C. Peng, X. Chen, L. Tagliacozzo, G. Su, and M. Lewenstein, arXiv:1708.09213.

[7] A. Pelissetto and E. Vicari, Phys. Rep. 368, 549 (2002).

[8] A. A. Belavin, A. M. Polyakov, and A. B. Zamolodchikov, Nucl. Phys. B B241, 333 (1984).

[9] M. Henkel, Conformal Invariance and Critical Phenomena, Theoretical and Mathematical Physics (Springer-Verlag, Berlin, 1999).

[10] Edited by J. L. Cardy, Finite-Size Scaling, Current PhysicsSources and Comments Vol. 2 (Elsevier, Amsterdam, 1988).

[11] L. Tagliacozzo, T. R. de Oliveira, S. Iblisdir, and J. I. Latorre, Phys. Rev. B 78, 024410 (2008).

[12] C. Holzhey, F. Larsen, and F. Wilczek, Nucl. Phys. B 424, 443 (1994).

[13] P. Calabrese and J. Cardy, J. Stat. Mech.: Theory Exp. (2004) P06002.

[14] G. Vidal, J. I. Latorre, E. Rico, and A. Kitaev, Phys. Rev. Lett. 90, 227902 (2003).

[15] C. Callan and F. Wilczek, Phys. Lett. B 333, 55 (1994).

[16] P. Calabrese, J. Cardy, and E. Tonni, J. Stat. Mech.: Theory Exp. (2009) P11001.

[17] P. Calabrese, J. Cardy, and E. Tonni, J. Stat. Mech.: Theory Exp. (2011) P01021.

[18] V. Alba, L. Tagliacozzo, and P. Calabrese, J. Stat. Mech.: Theory Exp. (2011) P06012.

[19] A. Coser, L. Tagliacozzo, and E. Tonni, J. Stat. Mech.: Theory Exp. (2014) P01008.

[20] C. De Nobili, A. Coser, and E. Tonni, J. Stat. Mech.: Theory Exp. (2015) P06021.

[21] H. Li and F. D. M. Haldane, Phys. Rev. Lett. 101, 010504 (2008).

[22] I. Peschel and T. T. Truong, Z. Phys. B 69, 385 (1987).

[23] P. Calabrese and A. Lefevre, Phys. Rev. A 78, 032329 (2008).

[24] D. Poilblanc, Phys. Rev. Lett. 105, 077202 (2010).

[25] J. I. Cirac, D. Poilblanc, N. Schuch, and F. Verstraete, Phys. Rev. B 83, 245134 (2011).

[26] V. Alba, M. Haque, and A. M. Läuchli, Phys. Rev. Lett. 108, 227201 (2012).

[27] V. Alba, M. Haque, and A. M. Läuchli, J. Stat. Mech.: Theory Exp. (2012) P08011.

[28] V. Alba, M. Haque, and A. M. Läuchli, Phys. Rev. Lett. 110, 260403 (2013).

[29] J. Dubail, N. Read, and E. H. Rezayi, Phys. Rev. B 85, 115321 (2012).

[30] A. M. Läuchli, arXiv:1303.0741.

[31] J. Cardy and E. Tonni, J. Stat. Mech.: Theory Exp. (2016) 123103.

[32] J. Cardy, Phys. Rev. Lett. 106, 150404 (2011).

[33] D. A. Abanin and E. Demler, Phys. Rev. Lett. 109, 020504 (2012).

[34] A. J. Daley, H. Pichler, J. Schachenmayer, and P. Zoller, Phys. Rev. Lett. 109, 020505 (2012).
[35] R. Islam, R. Ma, P. M. Preiss, M. Eric Tai, A. Lukin, M. Rispoli, and M. Greiner, Nature (London) 528, 77 (2015).

[36] P. Hauke, M. Heyl, L. Tagliacozzo, and P. Zoller, Nat. Phys. 12, 778 (2016).

[37] H. Pichler, G. Zhu, A. Seif, P. Zoller, and M. Hafezi, Phys. Rev. X 6, 041033 (2016).

[38] A. M. Kaufman, M. E. Tai, A. Lukin, M. Rispoli, R. Schittko, P. M. Preiss, and M. Greiner, Science 353, 794 (2016).

[39] M. Dalmonte, B. Vermersch, and P. Zoller, Nat. Phys. 14, 827 (2018).

[40] A. Elben, B. Vermersch, M. Dalmonte, J. I. Cirac, and P. Zoller, Phys. Rev. Lett. 120, 050406 (2018).

[41] B. Vermersch, A. Elben, M. Dalmonte, J. I. Cirac, and P. Zoller, Phys. Rev. A 97, 023604 (2018).

[42] T. Brydges, A. Elben, P. Jurcevic, B. Vermersch, C. Maier, B. P. Lanyon, P. Zoller, R. Blatt, and C. F. Roos, Science 364, 260 (2019).

[43] A. Lukin, M. Rispoli, R. Schittko, M. E. Tai, A. M. Kaufman, S. Choi, V. Khemani, J. Léonard, and M. Greiner, Science 364, 256 (2019).

[44] P. Calabrese and J. Cardy, J. Stat. Mech.: Theory Exp. (2005) P04010.

[45] G. De Chiara, S. Montangero, P. Calabrese, and R. Fazio, J. Stat. Mech.: Theory Exp. (2006) P03001.

[46] A. Nahum, J. Ruhman, S. Vijay, and J. Haah, Phys. Rev. X 7, 031016 (2017).

[47] G. Vidal, Phys. Rev. Lett. 93, 040502 (2004).

[48] S. R. White and A. E. Feiguin, Phys. Rev. Lett. 93, 076401 (2004).

[49] A. J. Daley, C. Kollath, U. Schollwöck, and G. Vidal, J. Stat. Mech.: Theory Exp. (2004) P04005.

[50] S. Paeckel, T. Köhler, A. Swoboda, S. R. Manmana, U. Schollwöck, and C. Hubig, Ann. Phys. 411, 167998 (2019).

[51] E. Leviatan, F. Pollmann, J. H. Bardarson, D. A. Huse, and E. Altman, arXiv:1702.08894.

[52] C. D. White, M. Zaletel, R. S. K. Mong, and G. Refael, Phys. Rev. B 97, 035127 (2018).

[53] J. Surace, M. Piani, and L. Tagliacozzo, Phys. Rev. B 99, 235115 (2019).

[54] M. M. Rams and M. Zwolak, Phys. Rev. Lett. 124, 137701 (2020).

[55] C. Krumnow, J. Eisert, and Ö. Legeza, arXiv:1904.11999.

[56] D. Fausti, R. I. Tobey, N. Dean, S. Kaiser, A. Dienst, M. C. Hoffmann, S. Pyon, T. Takayama, H. Takagi, and A. Cavalleri, Science 331, 189 (2011).

[57] D. A. Abanin and Z. Papić, Ann. Phys. 529, 1700169 (2017).

[58] F. Alet and N. Laflorencie, C. R. Phys. 19, 498 (2018).

[59] M. Rigol, V. Dunjko, and M. Olshanii, Nature (London) 452, 854 (2008).

[60] A. Polkovnikov, K. Sengupta, A. Silva, and M. Vengalattore, Rev. Mod. Phys. 83, 863 (2011).

[61] J. Eisert, M. Friesdorf, and C. Gogolin, Nat. Phys. 11, 124 (2015).

[62] L. D’Alessio, Y. Kafri, A. Polkovnikov, and M. Rigol, Adv. Phys. 65, 239 (2016).

[63] M. Prüfer, P. Kunkel, H. Strobel, S. Lannig, D. Linnemann, C.-M. Schmied, J. Berges, T. Gasenzer, and M. K. Oberthaler, Nature (London) 563, 217 (2018). 
[64] M. Heyl, A. Polkovnikov, and S. Kehrein, Phys. Rev. Lett. 110, 135704 (2013).

[65] B. Žunkovič, M. Heyl, M. Knap, and A. Silva, Phys. Rev. Lett. 120, 130601 (2018).

[66] M. Heyl, Europhys. Lett. 125, 26001 (2019).

[67] See Supplemental Material at http://link.aps.org/ supplemental/10.1103/PhysRevB.101.241107 for detail on $g_{r} S_{A}$, the BCFT analysis, more examples of quenches, and GGE.

[68] P. Jordan and E. Wigner, Z. Phys. 47, 631 (1928).

[69] E. Lieb, T. Schultz, and D. Mattis, Ann. Phys. (NY) 16, 407 (1961).

[70] E. Barouch, B. M. McCoy, and M. Dresden, Phys. Rev. A 2, 1075 (1970).

[71] E. Barouch and B. M. McCoy, Phys. Rev. A 3, 786 (1971).

[72] E. Barouch and B. M. McCoy, Phys. Rev. A 3, 2137 (1971).

[73] G. D. Giulio, R. Arias, and E. Tonni, J. Stat. Mech.: Theory Exp. (2019) 123103.

[74] P. Calabrese and J. Cardy, Phys. Rev. Lett. 96, 136801 (2006).

[75] P. Calabrese and J. Cardy, J. Stat. Mech.: Theory Exp. (2007) P06008.

[76] D. W. Robinson, J. Aust. Math. Soc. Series B, Appl. Math. 19, 387 (1976).

[77] O. Bratteli and D. W. Robinson, Operator Algebras and Quantum Statistical Mechanics II: Equilibrium States Models in Quantum Statistical Mechanics, Theoretical and Mathematical Physics (Springer-Verlag, Berlin, 1981).

[78] J. Cardy, Phys. Rev. Lett. 112, 220401 (2014).

[79] J. Cardy, J. Phys. A 49, 415401 (2016).

[80] T. Takayanagi and T. Ugajin, J. High Energy Phys. 11 (2010) 054.

[81] E. da Silva, E. Lopez, J. Mas, and A. Serantes, J. High Energy Phys. 04 (2015) 38

[82] J. Cardy, Nucl. Phys. B 275, 200 (1986).

[83] J. Cardy, Nucl. Phys. B 324, 581 (1989).
[84] P. Calabrese and J. Cardy, J. Stat. Mech.: Theory Exp. (2016) 064003.

[85] I. Peschel, J. Stat. Mech.: Theory Exp. (2004) P06004.

[86] K. Ohmori and Y. Tachikawa, J. Stat. Mech.: Theory Exp. (2015) P04010.

[87] V. Alba, P. Calabrese, and E. Tonni, J. Phys. A 51, 024001 (2017).

[88] E. Tonni, J. Rodríguez-Laguna, and G. Sierra, J. Stat. Mech.: Theory Exp. (2018) 043105.

[89] X. Wen, S. Ryu, and A. W. W. Ludwig, J. Stat. Mech.: Theory Exp. (2018) 113103.

[90] J. Cardy, J. Stat. Mech. Theory Exp. (2016) 023103.

[91] G. Evenbly, R. N. C. Pfeifer, V. Picó, S. Iblisdir, L. Tagliacozzo, I. P. McCulloch, and G. Vidal, Phys. Rev. B 82, 161107(R) (2010).

[92] G. Evenbly and G. Vidal, J. Stat. Phys. 157, 931 (2014).

[93] A. Polkovnikov and M. Rigol (private communication).

[94] G. Torlai, L. Tagliacozzo, and G. De Chiara, J. Stat. Mech.: Theory Exp. (2014) P06001.

[95] M. Fagotti, arXiv:1308.0277.

[96] J. Surace, L. Tagliacozzo, and E. Tonni (unpublished).

[97] J. J. Bisognano and E. H. Wichmann, J. Math. Phys. 16, 985 (1975).

[98] I. Peschel and M.-C. Chung, J. Phys. A 32, 8419 (1999).

[99] I. Peschel, J. Phys. A 36, L205 (2003).

[100] I. Peschel and V. Eisler, J. Phys. A: Math. Theor. 42, 504003 (2009).

[101] H. Casini, M. Huerta, and R. C. Myers, J. High Energy Phys. 05 (2011) 036.

[102] V. Eisler and I. Peschel, J. Phys. A 50, 284003 (2017).

[103] V. Eisler, E. Tonni, and I. Peschel, J. Stat. Mech.: Theory Exp. (2019) 073101.

[104] G. D. Giulio and E. Tonni, J. Stat. Mech. Theory Exp. (2020) 033102.

[105] M. Fagotti and F. H. L. Essler, Phys. Rev. B 87, 245107 (2013). 